\title{
Strontium coating by electrochemical deposition improves implant osseointegration in osteopenic models
}

\author{
YONGQIANG LIANG ${ }^{1,2}$, HAOYAN LI $^{1}, \mathrm{JIANG} \mathrm{XU}^{3}, \mathrm{XIN} \mathrm{LI}^{1}, \mathrm{XINCHANG} \mathrm{LI}^{1}$, \\ YUTING YAN $^{1}$, MENGCHUN QI ${ }^{1}$ and $\mathrm{MIN} \mathrm{HU}^{2}$ \\ ${ }^{1}$ College of Stomatology, Hebei United University, Tangshan, Hebei 063000; \\ ${ }^{2}$ Department of Stomatology, Chinese PLA General Hospital, Beijing 100853; \\ ${ }^{3}$ Department of Stomatology, People's Hospital of Tongchuan, Shaanxi 727000, P.R. China
}

Received March 22, 2014; Accepted October 3, 2014

DOI: $10.3892 / \mathrm{etm} .2014 .2038$

\begin{abstract}
Osteopenia, a preclinical state of osteoporosis, restricts the application of adult orthodontic implant anchorage and tooth implantation. Strontium (Sr) is able to promote bone formation and inhibit bone absorption. The aim of the present study was to evaluate a new method for improving the success rate of dental implantation. In this study, an electrochemical deposition (ECD) method was used to prepare a Sr coating on a titanium implant. The coating composition was investigated by energy dispersive X-ray spectroscopy and X-ray diffraction, and the surface morphology of the coating was studied using scanning electron microscopy. A total of 24 Sprague-Dawley rats received bilateral ovariectomy $(\mathrm{OVX})$ and an additional 12 rats underwent a sham surgery. All rats were then implanted in the bilateral tibiae with titanium mini-implants with or without a $\mathrm{Sr}$ coating. The results of histological examination and a fluorescence double labeling assay showed strong new bone formation with a wider zone between the double labels, a higher rate of bone mineralization and better osseointegration in the OVX rats that received Sr-coated implants compared with the OVX rats that received uncoated implants. The study indicates that Sr coatings are easily applied by an ECD method, and that Sr coatings have a promoting effect on implant osseointegration in animals with osteopenia.
\end{abstract}

\section{Introduction}

Osteoporosis is a major factor restricting adult orthodontic implant anchorage and tooth implantation. Previous studies have shown that osteoporosis affects the jawbone and weakens the osseointegration ability of dental implants (1-3).

Correspondence to: Professor Min Hu, Department of Stomatology, Chinese PLA General Hospital, 28 Fuxing Road, Haidian, Beijing 100853, P.R. China

E-mail: humin301@gmail.com

Key words: strontium, coating, implant, osteopenia, osseointegration
A reduction of bone mineral density of between 1 and 2.5 standard deviations is defined as osteopenia. Prior to osteoporosis, the human body experiences a pathological process in which bone mineral density is decreased. Osteopenia is a preclinical state of osteoporosis (4). Therefore, osteopenia is considered as a risk factor against the success of implantation.

The conventional treatment method for osteoporosis is drug therapy to prevent bone absorption. However, this modality is associated with adverse effects, drug resistance and long-term risks (5-7). Strontium ( $\mathrm{Sr}$ ) is an essential trace element for human skeletal components. It can promote bone formation and inhibit bone absorption (8). In vitro and in vivo assays have demonstrated that $\mathrm{Sr}$ can promote osteoblast proliferation and inhibit osteoclast proliferation, and in vitro experiments have indicated that $\mathrm{Sr}$ has a positive role in bone tissue engineering (9).

In the present study, Sr coatings were deposited on the surface of titanium implants by electrochemical deposition (ECD) and the implants were evaluated in an ovariectomized rat model. The aim of the study was to identify a new method for improving the success rate of dental implantation.

\section{Materials and methods}

Preparation of $\mathrm{Sr}$ coating by ECD. Commercial grade 4 unalloyed titanium plates $(10 \times 10 \times 2 \mathrm{~mm})$ and custom-made screw-type titanium implants $(1.5 \mathrm{~mm}$ diameter, $6.5 \mathrm{~mm}$ length; $99.8 \%$ Ti; National Engineering Research Center in Biomaterials, Sichuan University, Chengdu, China) were used as substrates for ECD. Prior to the deposition of coating, the titanium plates and implants were polished with up to 2,000 grid, immersed into hydrochloric acid and calcium chloride solutions successively, ultrasonically cleaned in acetone for $10 \mathrm{~min}$, rinsed with deionized water and air dried. The ECD experimental setup used for this study was a two-electrode cell configuration, as previously described (10). The working electrode (the coating substrate) was the titanium plate and the counter electrode was a platinum mesh. The distance between the electrodes was $2 \mathrm{~cm}$. The constant voltage was $2.5 \mathrm{~V}$, the temperature was $60^{\circ} \mathrm{C}$ and the reaction time was $1 \mathrm{~h}$. The electrolytes were $\mathrm{NH}_{4} \mathrm{H}_{2} \mathrm{PO}_{4} 0.036 \mathrm{~mol} / 1, \mathrm{CaCl}_{2}+\mathrm{SrCl}_{2} 0.06 \mathrm{~mol} / 1$ and $\mathrm{NaCl}_{2} 0.1 \mathrm{~mol} / 1, \mathrm{pH} 4.5$. 
Morphology and characterization of coating. The phase composition of the coating was analyzed by X-ray diffraction (XRD) using a D/MAX2500PC automatic XRD apparatus (Rigaku, Tokyo, Japan). The surface morphology and coating thickness were examined by scanning electron microscopy (SEM) using an S-4800 field emission scanning electron microscope (Hitachi, Tokyo, Japan). SEM was coupled with energy dispersive X-ray spectroscopy using a Noran ${ }^{\mathrm{TM}}$ system $7 \mathrm{X}$-ray microanalysis system (Thermo Fisher, Madison, WI, USA), which was used to determine the elemental composition of the biomimetic coating.

Animals and ovariectomy $(O V X)$. A total of 36 adult female Sprague Dawley (SD) rats, aged 3 months, were obtained from the Military Medical Science Academy of the PLA (Beijing, China). The in vivo study was approved by the Animal Care and Use Committee of Hebei United University [Tangshan, China; License no. SCXK (Jun 2009-003)]. Bilateral OVX was performed in 24 rats through an incision in the back under general anesthesia with an intraperitoneal injection of $10 \%$ chloral hydrate at a dose of $3 \mathrm{ml} / \mathrm{kg}$ (Chemical Reagent Co., Shanghai, China), while the remaining animals (12 rats) underwent a sham surgery in which the ovaries were examined and returned to the original position under the same protocol.

Implantation and animal grouping. Five weeks following OVX or sham surgery, all animals received simultaneous bilateral tibiae implantation using pure screw-shaped titanium implants with an ECD-generated Sr coating. The 12 animals that underwent a sham surgery were designated as the sham group, and the animals that underwent OVX were randomly divided into two groups, with 12 animals/group. One group received implants with a $\mathrm{Sr}$ coating ( $\mathrm{Sr}$ group) and the other received implants without an $\mathrm{Sr}$ coating (OVX group).

Fluorescent labeling. Following implantation, the SD rats were subdermally injected once a day with tetracycline hydrochloride at $30 \mathrm{mg} / \mathrm{kg}$ on days 13 and 14 and calcein at $6 \mathrm{mg} / \mathrm{kg}$ on days 3 and 4.

Histological examination. All rats were sacrificed at 4 weeks following implantation. Specimens of tibiae were fixed in $10 \%$ neutral buffered formalin for 5 days, dehydrated in increasing gradients of alcohol, and embedded in methylmethacrylate resin. Undecalcified, ground $30-\mu \mathrm{m}$ sections parallel to the long axis of the implant and vertical to the long axis of tibiae were obtained using an Exakt saw and grinding equipment (Exakt 300; Exact Advanced Technologies, GmbH, Norderstedt, Germany). The sections were stained with toluidine blue. Three sections of each specimen were examined at a magnification of $\mathrm{x} 100$.

Histomorphometric analysis was performed with a semi-automated digitizing image analysis system, comprising a Nikon ECLIPSE E600 stereomicroscope, a computer-coupled Nikon DXM1200 Digital Camera and NIS-Elements F 2.20 image software (Nikon Corporation, Tokyo, Japan). Histomorphometric indices included the implant-bone contact rate (IBCR), defined as the direct implant-bone interface to total implant surface, and the ratio of calcified bone volume to total bone volume (BV/TV) within $2.0 \mathrm{~mm}$ of the axis of the implant. In each section, five equally spaced sites of each screw were measured and the mean value of all screws was accepted as the value of the index of the section (11).

Bone dynamic indices. The distance between double labels (DDL), mineral apposition rate (MAR) and mineralizing surface/bone surface ratio (MS/BS) were calculated. The MAR was determined by measuring the distance between the two fluorescent lines. In each field, five equally distributed sites of each double fluorescent line were measured and the mean of the values in the field was accepted as the value of the field. The measurements were conducted with a confocal microscope (FV1000; Olympus Corporation, Tokyo, Japan). The MS/BS value was calculated as follows: MS/BS $(\%)=(0.5$ single labeled perimeter + double labeled perimeter)/bone perimeter $\mathrm{x} 100$.

Biomechanical testing. The removal torque of the implants was examined with a method similar to that previously reported (12). Briefly, the specimens were fixed in $10 \%$ neutral buffered formalin and embedded in a quadrate metal box with dental plaster. The testing equipment was a force measuring device (Asida DZE-5; Zhengye Electronics Co., Ltd., Dongguan, China) for recording the peak force value in Newtons (N) required to loosen the implant and a specially made wrench that connected the implant and the force measuring device. The implant used was specially designed with a square cap suitable for application of the wrench. The removal torque was calculated by multiplying the peak force value with the distance between the force point and the center of the implant.

Statistical analysis. Data are expressed as mean \pm standard deviation (SD) and statistical analyses were performed using SPSS software, version 12.0 (SPSS, Inc., Chicago, IL, USA). One-way analysis of variance (ANOVA) was used to assess differences between OVX and sham-operated rats. $\mathrm{P}<0.05$ was considered to be indicate a statistically significant difference.

\section{Results}

Coating XRD analysis. XRD analysis indicated that the coating comprised strontium hydrogen phosphate $\left(\mathrm{SrHPO}_{4}\right)$. The relative intensities of the diffraction peaks for the $\mathrm{SrHPO}_{4}$ crystals (102) were higher than those for other crystal face intensities, and revealed a (102) crystal face growth advantage (Fig. 1).

Analysis of the morphology of the coating surface. The $\mathrm{Sr}$ coating thickness analyzed by SEM was $25 \mu \mathrm{m}$. The Sr coating was observed to consist of lamellar crystals in aggregated clusters or a petal-like arrangement (Fig. 2).

Histological examination. Histological images obtained from undecalcified sections are shown in Fig. 3. In the OVX group, bone formation was diminished with poor bone continuity. By contrast, in the Sr group, new bone formation was evident with new thicker laminar bones. Certain laminar bones were discontinuous. Bone formation was normal in the sham group, with prominent laminar bone. Table I shows various histomor- 


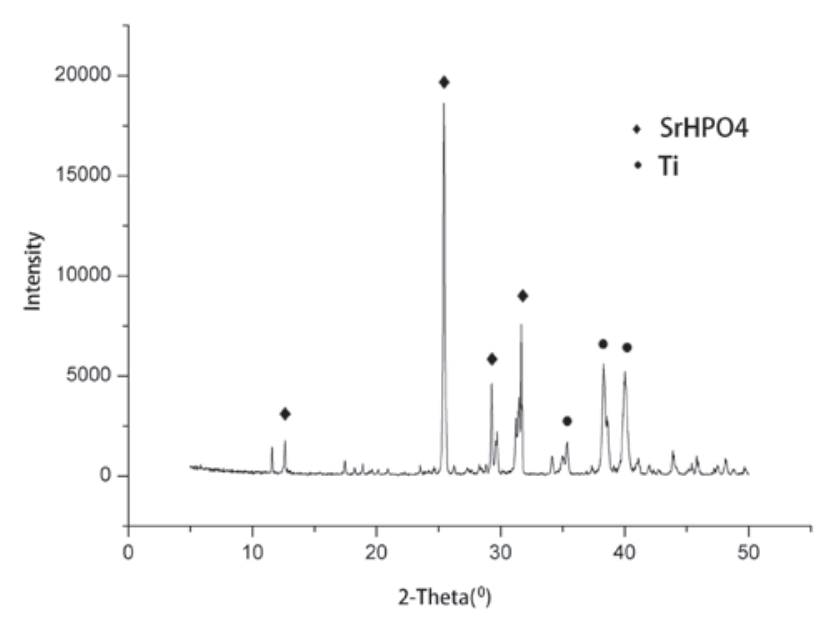

Figure 1. X-ray diffraction analysis of the strontium coating. The results indicate that the coating comprised strontium hydrogen phosphate.

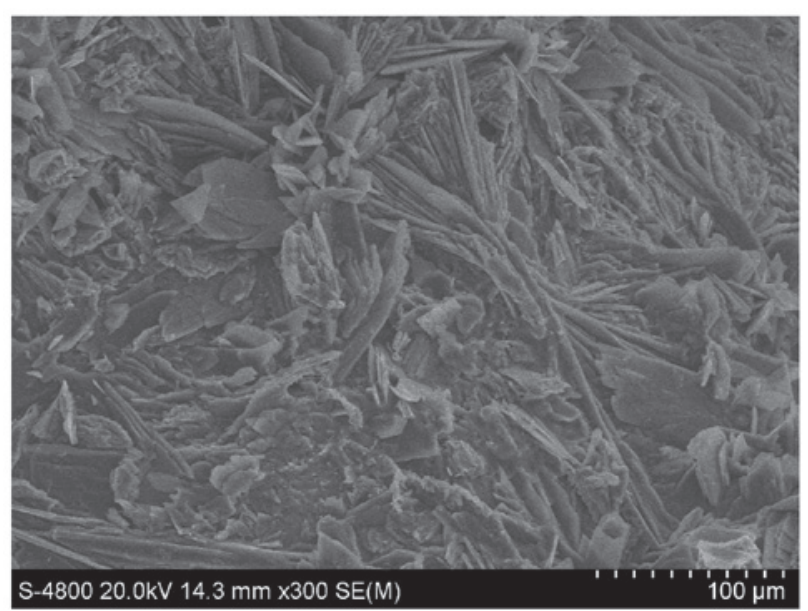

Figure 2. Morphology of the strontium ( $\mathrm{Sr}$ ) coating by scanning electron microscopy. The $\mathrm{Sr}$ coating consists of lamellar crystals in aggregated clusters or a petal-like arrangement.

phometric indices, namely the IBCR, BV/TV and values for the thickness of the bone lamellar interface (T). The IBCR, $\mathrm{BV} / \mathrm{TV}$ and $\mathrm{T}$ in the $\mathrm{Sr}$ group were significantly higher than those of the OVX group $(\mathrm{P}<0.01)$, and the OVX group had the lowest IBCR, BV/TV and $\mathrm{T}(\mathrm{P}<0.05$ or $\mathrm{P}<0.01)$.

Fluorescent histology. Confocal microscopy showed strong fluorescent labeling in the OVX and $\mathrm{Sr}$ groups, mainly at the trabecular bone surface and at the interface between the implant and bone (Fig. 4). The sham group showed strong fluorescent intensity, with thick and continuous fluorescently labeled lines, and a wide distance between the two fluorescent lines. However, in the OVX group, the fluorescence intensity was weak, the fluorescently labeled lines were thin and discontinuous, and the distance between the two fluorescent lines was narrow. The appearance of fluorescent labeling was markedly strengthened in the $\mathrm{Sr}$ group and was greater than that in the OVX group, although it was not as strong as that in the sham group. The aforementioned changes were also supported by quantitative analysis. The highest bone dynamic indices, specifically DDL, MAR and MS/BS values, were
Table I. Histomorphometric analysis of bone indices among the three groups $(\mathrm{n}=12)$.

\begin{tabular}{llll}
\hline Group & IBCR $(\%)$ & \multicolumn{1}{c}{ BV/TV $(\%)$} & \multicolumn{1}{c}{ T $(\mu \mathrm{m})$} \\
\hline Sham & $62.71 \pm 4.60$ & $59.24 \pm 5.13$ & $67.01 \pm 6.66$ \\
OVX & $39.34 \pm 4.42^{\mathrm{a}}$ & $42.39 \pm 5.48^{\mathrm{a}}$ & $49.34 \pm 4.49^{\mathrm{a}}$ \\
Sr & $58.72 \pm 3.85^{\mathrm{b}, \mathrm{c}}$ & $49.39 \pm 7.14^{\mathrm{a}, \mathrm{d}}$ & $54.27 \pm 6.95^{\mathrm{a}, \mathrm{c}}$ \\
\hline
\end{tabular}

IBCR, implant-bone contact rate; BV/TV, calcified bone volume/total bone volume; $\mathrm{T}$, thickness of the lamellar bone interface; OVX, ovariectomy. Data presented are the mean \pm standard deviation. ${ }^{\mathrm{a}} \mathrm{P}<0.01$ compared with the sham group; ${ }^{b} \mathrm{P}<0.05$ compared with sham group; ${ }^{\mathrm{c}} \mathrm{P}<0.01$ compared with the OVX group; ${ }^{\mathrm{d}} \mathrm{P}<0.05$ compared with the OVX group.

Table II. Dynamic indices of bone metabolism $(n=12)$.

\begin{tabular}{llcl}
\hline Group & DDL $(\mu \mathrm{m})$ & MAR $(\mu \mathrm{m} /$ day $)$ & MS/BS \\
\hline Sham & $34.99 \pm 3.53$ & $2.72 \pm 0.20$ & $0.28 \pm 0.05$ \\
OVX & $20.59 \pm 3.13^{\mathrm{a}}$ & $1.61 \pm 0.30^{\mathrm{a}}$ & $0.16 \pm 0.03^{\mathrm{a}}$ \\
Sr & $30.41 \pm 3.14^{\mathrm{a}, \mathrm{b}}$ & $2.30 \pm 0.40^{\mathrm{b}, \mathrm{c}}$ & $0.20 \pm 0.03^{\mathrm{c}, \mathrm{d}}$ \\
\hline
\end{tabular}

DDL, distance between double labels; MAR, mineral apposition rate; MS/BS, mineralizing surface/bone surface ratio; OVX, ovariectomy. Data presented are the mean \pm standard deviation. ${ }^{a} \mathrm{P}<0.01$ compared with the sham group; ${ }^{\mathrm{b}} \mathrm{P}<0.01$ compared with the OVX group; ${ }^{\mathrm{c}} \mathrm{P}<0.05$ compared with the sham group; ${ }^{\mathrm{d}} \mathrm{P}<0.01$ compared with the OVX group.

found in the sham group, followed in turn by the Sr and OVX groups $(\mathrm{P}<0.05$ or $\mathrm{P}<0.01)$. Significant differences in these indices were also observed between the $\mathrm{Sr}$ and OVX groups $(\mathrm{P}<0.05$ or $\mathrm{P}<0.01)$. Thus, the $\mathrm{Sr}$ group exhibited active bone metabolism (Table II).

Biomechanical test. The removal torques of the titanium implants in the sham, OVX and $\mathrm{Sr}$ groups were 27.94 \pm 1.43 , $22.04 \pm 2.11$ and $25.30 \pm 1.38$ N.cm respectively. The removal torques in the OVX and $\mathrm{Sr}$ groups were significantly increased when compared with that of the sham group $(\mathrm{P}<0.01)$. The removal torque in the $\mathrm{Sr}$ group was higher than that in the OVX group $(\mathrm{P}<0.01)$.

\section{Discussion}

Osteoporosis has brought many challenges to clinicians. Osteoporosis and osteopenia restrict orthodontic implant anchorage and tooth implantation in adults. A previous study showed that implant failure in patients with osteoporosis is associated with the thickness of cortical bone (13). In the present study, it was found that new bone formation was increased around the Sr coating, and implant osseointegration was promoted. This suggests that Sr may improve the stability of implants.

Implant osseointegration in patients with osteoporosis is affected by reduced bone mass and bone tissue microstruc- 
A

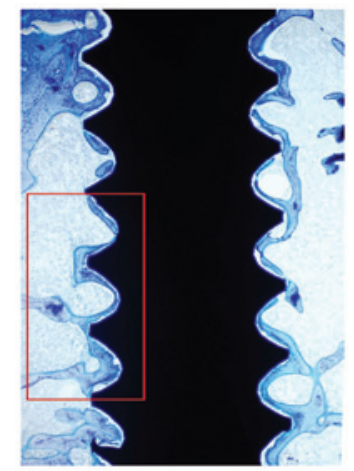

D

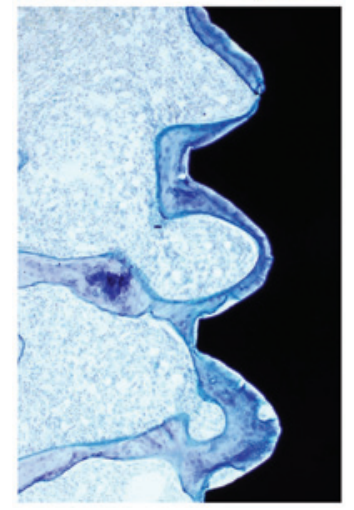

B

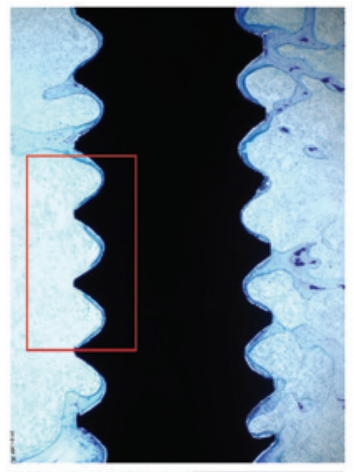

$\mathbf{E}$

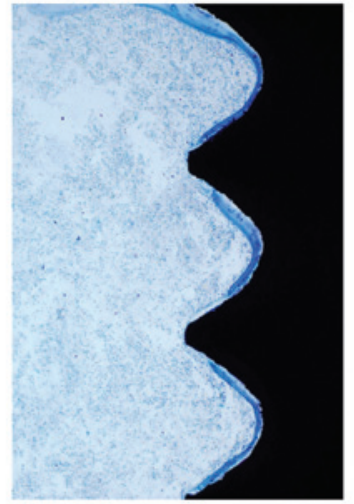

C

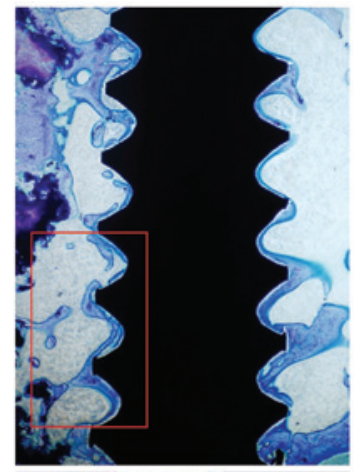

F

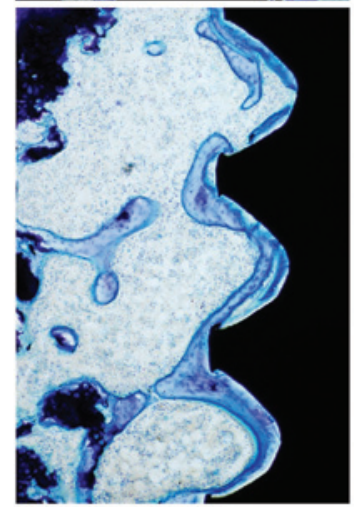

Figure 3. Histological examination of the proximal tibia with implants and bone grafts with toluidine blue staining. Images for (A,D) the sham group, $(\mathrm{B}, \mathrm{E})$ OVX group and $(\mathrm{C}, \mathrm{F})$ Sr group. The OVX group had the least implant-bone integration and calcified bone, while the Sr-coating group had a marked increase of implant-bone integration and calcified bone. Magnification (A-C), x40; (D-F), x100. OVX, ovariectomized; Sr, strontium.
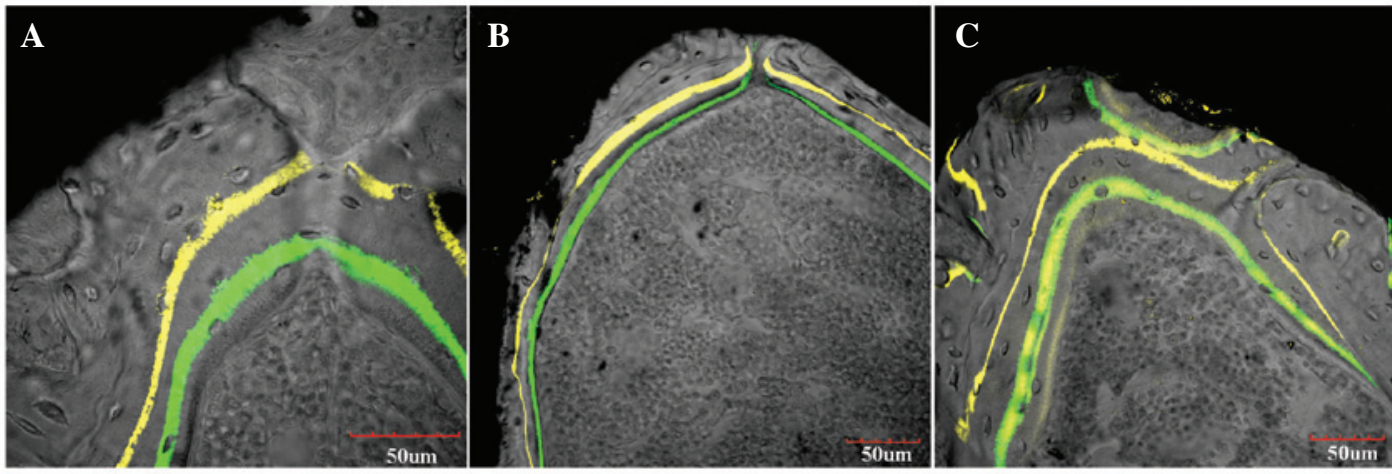

Figure 4. Fluorescent double labeling in the (A) sham, (B) OVX and (C) Sr groups 4 weeks post-implantation as observed by laser scanning confocal microscopy. The OVX group showed the poorest fluorescent labeling, in which the fluorescent intensity was weak, the fluorescent lines were thin and discontinuous, and the distance between the double-labeled line was narrow. Magnfication, x400. OVX, ovariectomized; Sr, strontium.

tural changes (3). However, Motohashi et al discovered that bone formation and distribution around the implant in rats with osteoporosis are the same as those in normal rats in the early stage of osteoporosis; however the absorption rate of new bone is accelerated in the later stage of osteoporosis (14). The studies conducted by Mori et al (15) and Fujimoto et al (16) demonstrated that new bone formation around the implant in osteoporotic animals was delayed compared with that in healthy animals. Moreover, osteoporosis affects bone healing around the implant. Due to a reduction of cancellous bone in patients with osteoporosis, these patients require more frequent monitoring of implant stability.

After the implant is implanted in bone, the implant is not able to bear the orthodontic force if healthy bone is lacking around the mini-implant (17). The success of a mini-implant is determined by the stability of the connection between the implant and the bone tissue. Reduced estrogen levels can accelerate the bone metabolic rate and result in decreased quality and density of bone, which affects the initial stability of the implant (18). Osteopenia can develop into osteoporosis (19). Therefore, the timely intervention of osteopenia is necessary for the management of osteoporosis.

$\mathrm{Sr}$ reduces bone resorption by inhibiting the proliferation of osteoclasts and improving bone formation by stimulating the proliferation of osteoblasts (20). Sr has also been shown to enhance osteogenic cell replication and the activity of osteoblasts, including the synthesis of bone matrix and the activity of alkaline phosphatase, in addition to reducing 
osteoclast markers generated in the differentiation of bone marrow cells, inhibiting the differentiation of osteoclasts and reducing the activity of osteoclasts $(21,22)$. Furthermore, Sr stimulates the osteogenic differentiation of bone marrow mesenchymal stem cells and other progenitor cells (23). Studies have confirmed that Sr-doped hydroxyapatite, calcium phosphate, calcium silicate, calcium sulfate, boron bioactive glass and other materials, promote bone tissue reconstruction and new bone formation (24-26). In the present study, $\mathrm{Sr}$ significantly enhanced bone formation in ovariectomized rats with Sr-coated implants. This implies that tooth implants with a $\mathrm{Sr}$ coating may be useful in osteopenic patients. However, the application of $\mathrm{Sr}$ coatings requires further investigation for optimization.

The Sr coatings were deposited on the surfaces of pure titanium implants by ECD. Four weeks after implantation of the Sr-coated implants into the tibiae of the rats with osteopenia, the histomorphometric indices IBCR, BV/TV and T in the Sr group were observed to be significantly higher than those of the OVX group. These changes were also supported by quantitative analysis. The bone dynamic indices DDL, MAR and MS/BS in the Sr group were significantly higher than those of the OVX group. The Sr group exhibited active bone metabolism. The histomorphometric indices and bone dynamic indices confirmed that the presence of $\mathrm{Sr}$ at the surface of the implant promoted osteoblast function and inhibited osteoclast function. Ultimately, it promoted osseointegration and increased the removal torque.

In conclusion, $\mathrm{Sr}$ coatings are easily made by ECD. They have a promoting effect on implant osseointegration in animals with osteopenia, and provide a new proposition for patients with osteoporosis who require dental implants.

\section{Acknowledgements}

The study was partly supported by a National Natural Science Foundation of China (no. 81270965) and the Beijing Natural Science Foundation (7112124).

\section{References}

1. Akca K, Sarac E, Baysal U, Fanuscu M, Chang TL and Cehreli M Micro-morphologic changes around biophysically-stimulated titanium implants in ovariectomized rats. Head Face Med 3: 28, 2007.

2. Goldhahn J, Seebeck J, Frei R, Frenz B, Antoniadis I and Schneider E: New implant designs for fracture fixation in osteoporotic bone. Osteoporos Int 16 (Suppl 2): S112-S119, 2005.

3. Marco F, Milena F, Gianluca G and Vittoria O: Peri-implant osteogenesis in health and osteoporosis. Micron 36: 630-644, 2005.

4. Soen S, Fukunaga M, Sugimoto T, et al; Japanese Society for Bone and Mineral Research and Japan Osteoporosis Society Joint Review Committee for the Revision of the Diagnostic Criteria for Primary Osteoporosis: Diagnostic criteria for primary osteoporosis: year 2012 revision. J Bone Miner Metab 31: 247-257, 2013

5. Viera-Negrón YE, Ruan WH, Winger JN, Hou X, Sharawy MM and Borke JL: Effect of ovariectomy and alendronate on implant osseointegration in rat maxillary bone. J Oral Implantol 34 $76-82,2008$.
6. Du Z, Chen J, Yan F and Xiao Y: Effects of Simvastatin on bone healing around titanium implants in osteoporotic rats. Clin Oral Implants Res 20: 145-150, 2009.

7. Ohkawa Y, Tokunaga $\mathrm{K}$ and Endo N: Intermittent administration of human parathyroid hormone (1-34) increases new bone formation on the interface of hydroxyapatite coated titanium rods implanted into ovariectomized rat femora. J Orthop Sci 3: 533-542, 2008

8. Brennan TC, Rybchyn MS, Green W, Atwa S, Conigrave AD and Mason RS: Osteoblasts play key roles in the mechanisms of action of strontium ranelate. Br J Pharmacol 157: 1291-1300, 2009.

9. Fonseca JE: Rebalancing bone turnover in favour of formation with strontium ranelate: implications for bone strength. Rheumatology (Oxford) 47 (Suppl 4): iv17-iv19, 2008.

10. Le Nihouannen D, Hacking SA, Gbureck U, Komarova SV and Barralet JE: The use of RANKL-coated brushite cement to stimulate bone remodelling. Biomaterials 29: 3253-3259, 2008.

11. Qi MC, Zhou XQ, Hu J, et al: Oestrogen replacement therapy promotes bone healing around dental implants in osteoporotic rats. Int J Oral Maxillofac Surg 33: 279-285, 2004.

12. Qi M, Hu J, Li J, et al: Effect of zoledronate acid treatment on osseointegration and fixation of implants in autologous iliac bone grafts in ovariectomized rabbits. Bone 50: 119-127, 2012.

13. Herrmann I, Lekholm U, Holm S and Kultje C: Evaluation of patient and implant characteristics as potential prognostic factors for oral implant failures. Int J Oral Maxillofac Implants 20: 220-230, 2005

14. Motohashi M, Shirota T, Tokugawa Y, Ohno K, Michi K and Yamaguchi A: Bone reactions around hydroxyapatite-coated implants in ovariectomized rats. Oral Surg Oral Med Oral Pathol Oral Radiol Endod 87: 145-152, 1999.

15. Mori H, Manabe M, Kurachi Y and Nagumo M: Osseointegration of dental implants in rabbit bone with low mineral density. J Oral Maxillofac Surg 55: 351-362, 1997.

16. Fujimoto T, Niimi A, Sawai T and Ueda M: Effects of steroid-induced osteoporosis on osseointegration of titanium implants. Int J Oral Maxillofac Implants 13: 183-189, 1998.

17. Kang S, Lee SJ, Ahn SJ, Heo MS and Kim TW: Bone thickness of the palate for orthodontic mini-implant anchorage in adults. Am J Orthod Dentofacial Orthop 131 (4 Suppl): S74-S81, 2007.

18. Ohmae M, Saito S, Morohashi T, et al: A clinical and histological evaluation of titanium mini-implants as anchors for orthodontic intrusion in the beagle dog. Am J Orthod Dentofacial Orthop 119: 489-497, 2001.

19. Ozawa S, Ogawa T, Iida K, et al: Ovariectomy hinders the early stage of bone-implant integration: histomorphometric, biomechanical, and molecular analyses. Bone 30: 137-143, 2002.

20. Bonnelye E, Chabadel A, Saltel F and Jurdic P: Dual effect of strontium ranelate: stimulation of osteoblast differentiation and inhibition of osteoclast formation and resorption in vitro. Bone 42: 129-138, 2008.

21. Ni GX, Yao ZP, Huang GT, Liu WG and Lu WW: The effect of strontium incorporation in hydroxyapatite on osteoblasts in vitro. J Mater Sci Mater Med 22: 961-967, 2011.

22. Choudhary S, Halbout P, Alander C, Raisz L and Pilbeam C: Strontium ranelate promotes osteoblastic differentiation and mineralization of murine bone marrow stromal cells: involvement of prostaglandins. J Bone Miner Res 22: 1002-1010, 2007.

23. Capuccini C, Torricelli P, Sima F, et al: Strontium-substituted hydroxyapatite coatings synthesized by pulsed-laser deposition: in vitro osteoblast and osteoclast response. Acta Biomater 4: 1885-1893, 2008.

24. Caverzasio $\mathrm{J}$ and Thouverey C: Activation of FGF receptors is a new mechanism by which strontium ranelate induces osteoblastic cell growth. Cell Physiol Biochem 27: 243-250, 2011.

25. Baier M, Staudt P, Klein R, et al: Strontium enhances osseointegration of calcium phosphate cement: a histomorphometric pilot study in ovariectomized rats. J Orthop Surg Res 8: 16, 2013.

26. Pan HB, Zhao XL, Zhang X, et al: Strontium borate glass: potential biomaterial for bone regeneration. J R Soc Interface 7: 1025-1031, 2010. 